OPEN ACCESS

Edited by:

Matthias Tenbusch,

University Hospital Erlangen, Germany

Reviewed by:

Eun-Kyeong Jo,

Chungnam National University,

South Korea

Heinz Laubli,

University of Basel, Switzerland

*Correspondence:

Sha Tao

sha.tao@med.uni-duesseldorf.de

Specialty section:

This article was submitted to

Vaccines and Molecular Therapeutics,

a section of the journal

Frontiers in Immunology

Received: 13 December 2019

Accepted: 28 February 2020

Published: 19 March 2020

Citation:

Tao S and Drexler I (2020) Targeting Autophagy in Innate Immune Cells: Angel or Demon During Infection and Vaccination? Front. Immunol. 11:460

do: $10.3389 /$ fimmu.2020.00460

\section{Targeting Autophagy in Innate Immune Cells: Angel or Demon During Infection and Vaccination?}

\author{
Sha Tao* and Ingo Drexler \\ Institute for Virology, Düsseldorf University Hospital, Heinrich-Heine-University, Düsseldorf, Germany
}

Innate immune cells are the "doorkeepers" in the immune system and are important for the initiation of protective vaccine responses against infection. Being an essential regulatory component of the immune system in these cells, autophagy not only mediates pathogen clearance and cytokine production, but also balances the immune response by preventing harmful overreaction. Interestingly, recent literature indicates that autophagy is positively or negatively regulating the innate immune response in a cell type-specific manner. Moreover, autophagy serves as a bridge between innate and adaptive immunity. It is involved in antigen presentation by delivering pathogen compounds to $\mathrm{B}$ and $\mathrm{T}$ cells, which is important for effective immune protection. Upon infection, autophagy can also be hijacked by some pathogens for replication or evade host immune responses. As a result, autophagy seems like a double-edged sword to the immune response, strongly depending on the cell types involved and infection models used. In this review, the dual role of autophagy in regulating the immune system will be highlighted in various infection models with particular focus on dendritic cells, monocytes/macrophages and neutrophils. Targeting autophagy in these cells as for therapeutic application or prophylactic vaccination will be discussed considering both roles of autophagy, the "angel" enhancing innate immune responses, antigen presentation, pathogen clearance and dampening inflammation or the "demon" enabling viral replication and degrading innate immune components. A better understanding of this dual potential will help to utilize autophagy in innate immune cells in order to optimize vaccines or treatments against infectious diseases.

Keywords: autophagy, dendritic cells, macrophages, innate immunity, adaptive immunity, vaccines and therapies against infectious diseases

\section{INTRODUCTION}

The awarding of the Nobel Prize in 2016 to Yoshinori Ohsumi reflects the importance of autophagy in human health and disease. Autophagy is a homeostatic degradation process that enables cells to survive in case of stress, like accumulation of misfolded proteins and damaged organelles or starvation and energy deprivation. Mammalian cells deliver those "unwanted" materials to lysosomes for degradation. Three major ways can be distinguished: microautophagy, chaperonemediated autophagy, and macroautophagy. The latter has been intensively characterized in recent years because of its high impact on human health and disease (1). In this review, we will focus on macroautophagy, simply referred to as autophagy in the following. 
The autophagic pathway has been widely discussed and reviewed (1-3). Here, only a brief summary will be provided, including the four major steps in the pathway: initiation, membrane elongation, maturation/fusion and degradation. In mammalian cells, after a strong stimulus such as starvation, autophagosomes initiate as omegasomes at the endoplasmic reticulum (ER). Autophagy can be induced by two different arms of upstream signaling based on either mammalian target of rapamycin (mTOR) inactivation or adenosine monophosphate (AMP) activated protein kinase (AMPK) activation which leads to distinct Unc-51-like autophagy activating kinase 1 (ULK1) activation. mTOR typically responds to nutrient signals while AMPK responds to the energy status of the cell. Two protein complexes are important for phagophore formation: ULK and PI3K (phosphoinositide 3-kinase catalytic subunit type III) complex. The ULK complex consists of autophagyrelated (ATG)13, FIP200, ATG101, and ULK1. The PI3K complex comprises Beclin1, VPS34 (vacuolar protein sorting 34), VPS15 and ATG14L. Furthermore, two ubiqutin-like (UBL) conjugation complexes are important for the membrane extension. One is ATG16L1 complex, in which ATG12 is conjugated to ATG5 and then bind to ATG16L1. This facilitates another ubiquitin cascade involving ATG7 and ATG3, namely microtubule-associated protein 1A/1B-light chain 3 (LC3/ATG8) lipidation (conjugation to phosphatidylethanolamine-PE). LC3-PE mediates membrane tethering and fusion to extend the isolation membrane by recruiting membranes from multiple sources, leading to the formation of autophagosomes. During the final maturation, autophagosomes are decorated with Rab7 and tail-anchored SNAP receptor (SNARE) syntaxin 17 (STX17), which leads to the fusion with lysosomes and degradation of sequestered substrates (Figure 1). Recent findings suggest that autophagy can also occur in the absence of some key autophagy-related proteins (ATGs) through unconventional autophagy pathways, also called "non-canonical autophagy" (4-6). Furthermore, the double membrane does not necessarily elongate from a single source. Such variation gives alternatives to recognize or eliminate pathogens, for instance, receptor mediate internalization and LC3-associated phagocytosis (LAP).

The immune system is a big network with crosstalk of cells from innate and adaptive immunity. Autophagy is a key mechanism against invading bacteria, parasites, and viruses in innate immune cells including monocytes/macrophages, dendritic cells (conventional dendritic cells-cDCs and plasmacytoid dendritic cells-pDCs) and neutrophils. In the past few years, a number of studies have highlighted the potential

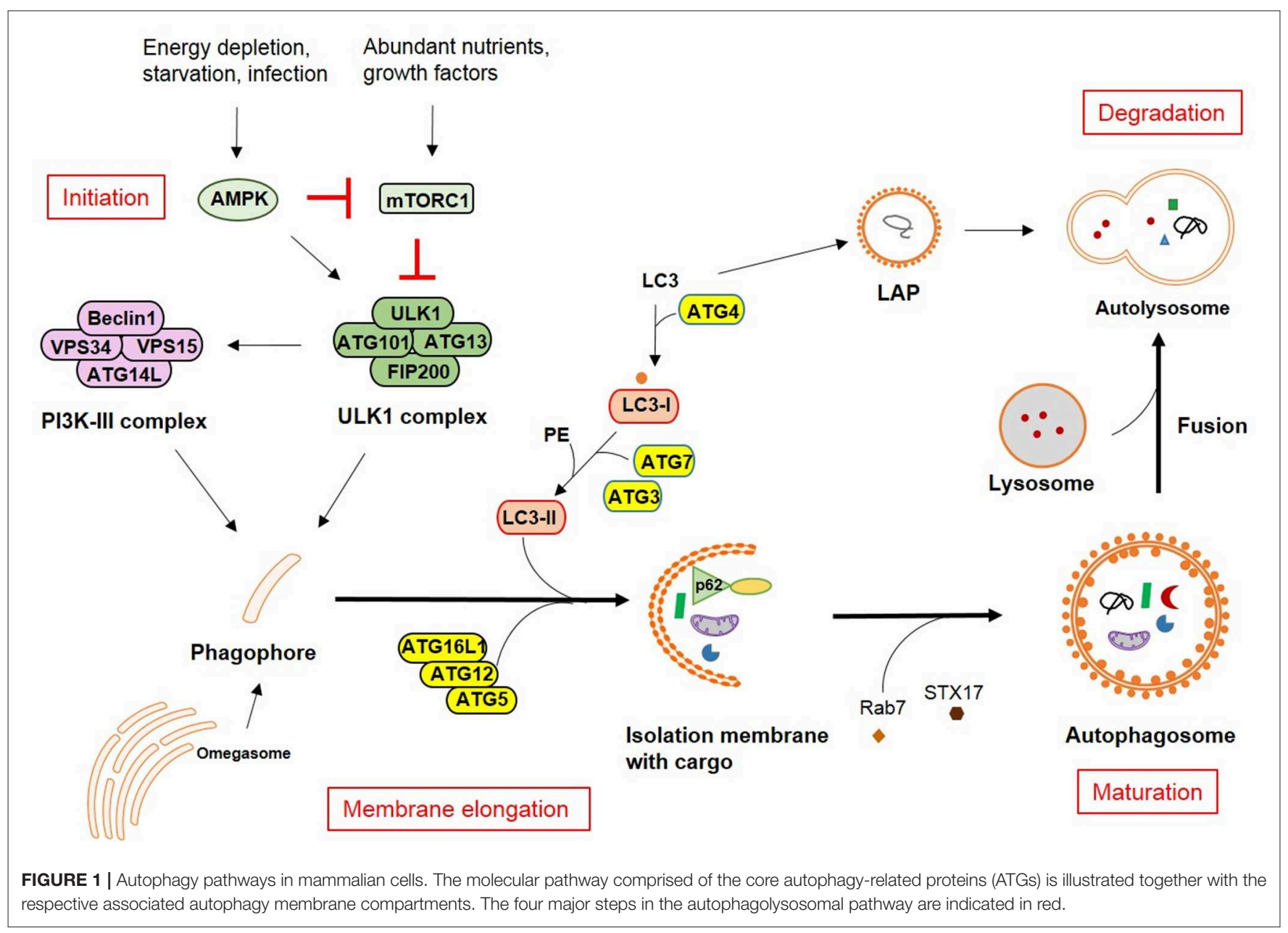


of targeting autophagy for the control of infections. These data combined with the emerging role of autophagy for immune impairment in some infectious diseases have attracted significant interest in developing autophagy modulators or targets as a new approach for vaccination.

\section{VACCINES TARGETING AUTOPHAGY FOR EFFECTIVE ANTIGEN PROCESSING AND PRESENTATION}

DCs as one of most potent professional antigen-presenting cells (APCs) bridge between the innate and adaptive immune system. They are particularly critical for naïve $\mathrm{T}$ cell activation and drive protective immunity against infection. An increasing number of recent studies have characterized the involvement of autophagy in various DC functions in physiological as well as pathological context $(7)$, especially with regard to $\mathrm{T}$ cell activation $(8,9)$ (Figure 2).

Autophagy has been shown to be involved in antigen processing and presentation in DCs, especially for MHC class II restricted peptides. Those peptides are often derived from lysosomal degradation, either from LAP (non-canonical pathway) (10) or from macroautophagy (canonical pathway). In the latter, cytosolic antigens are recognized by a group of SQSTM1-like receptors (SLRs) such as p62, NDP52, OPTN or NBR1. This selective form of autophagy is also called "xenophagy" $(3,11)$. SLRs serve as links to ubiquitin (tagged with substrates) and LC3 homologs on the autophagosomal membrane. Peptides are further loaded on MHC class II in late endosomal MHC class II containing compartments (MIIC) (12). LAP involves single-membraned phagosomes, but also leads to MHC class II presentation (5). As a result, it is not surprising that autophagy promoted MHC class II antigen presentation to CD4+ $\mathrm{T}$ cells in various infection models, such as modified vaccinia virus Ankara (13) or herpes simplex virus 1 (HSV-1) (14-17) or was able to enhance cytokine production by CD4+ $\mathrm{T}$ cells in Toxoplasma gondii ( $T$. gondii) (18) or respiratory syncytial virus (RSV) infection (19). Indeed, enabling access of antigens to autophagolysosomes by genetic engineering to link them to key components like LC3-II, greatly enhanced vaccine efficacy. For instance, human immunodeficiency virus-1 (HIV1) Gag and Env fail to colocalize with LC3 containing vesicles during infection. However, once antigens were targeted to LC3b, the autophagic degradation process was enhanced and could efficiently stimulate $\mathrm{CD} 4+\mathrm{T}$ cell responses $(20,21)$. Besides, autophagy also indirectly promoted antigen presentation by benefitting lysosomal enzyme activity during HIV-1 infection (22). Further examples include the conjugation of influenza A virus (IAV) matrix protein 1 (M1) to LC3 in DCs which led to enhanced antigen-specific CD4 $+\mathrm{T}$ cell responses (23). Japanese encephalitis (JEV) prM and E proteins fused to LC3 (pJME-LC3 DNA vaccine) allowed for increased $\mathrm{T}$ cell responses and long lasting antibody-mediated protection after immunization (24).

In addition to MHC class II presentation to $\mathrm{CD} 4+\mathrm{T}$ cells, autophagy in several APCs has been considered to contribute to MHC class I presentation to CD8+ T cells. Indeed, pDCs (25) as well as some subtypes of macrophages (26) showed potential for antigen capture and processing, and promoted $\mathrm{T}$ cell priming in infection models. Viral peptides derived from autophagosomes were further processed by proteasomes in HIV1 infected macrophages (27). The MHC class I presentation of human cytomegalovirus (HCMV) protein pUL138-derived peptide epitopes was autophagy-mediated and TAP-independent (28). H1N1 infected bone marrow-derived DCs (BMDCs) were activating strong $\mathrm{CD} 4+\mathrm{T}$ cell proliferation and additionally, were more efficiently cross-presenting antigen to $\mathrm{CD} 8+\mathrm{T}$ cells (29). All these reports suggest an interaction between vacuolar and MHC class I presentation pathways. Dribbles are autophagosomal structures derived from tumor cells after proteasomal inhibition and are currently tested as tools to enhance cross-presentation. Human DCs loaded with DRibbles isolated from tumor cells expressing CMV peptide epitopes were significantly more efficient in stimulating CD8+ memory $\mathrm{T}$ cells (30). Similarly, DCs loaded with DRibbles containing CMV proteins revealed a superior ability to induce CMV-specific $\mathrm{T}$ cells (31).

Among SLRs, p62 and NDP52 are considered most important for pathogen recognition through autophagy. p62 delivers ubiquitinated Mycobacterium tuberculosis (Mtb) proteins into

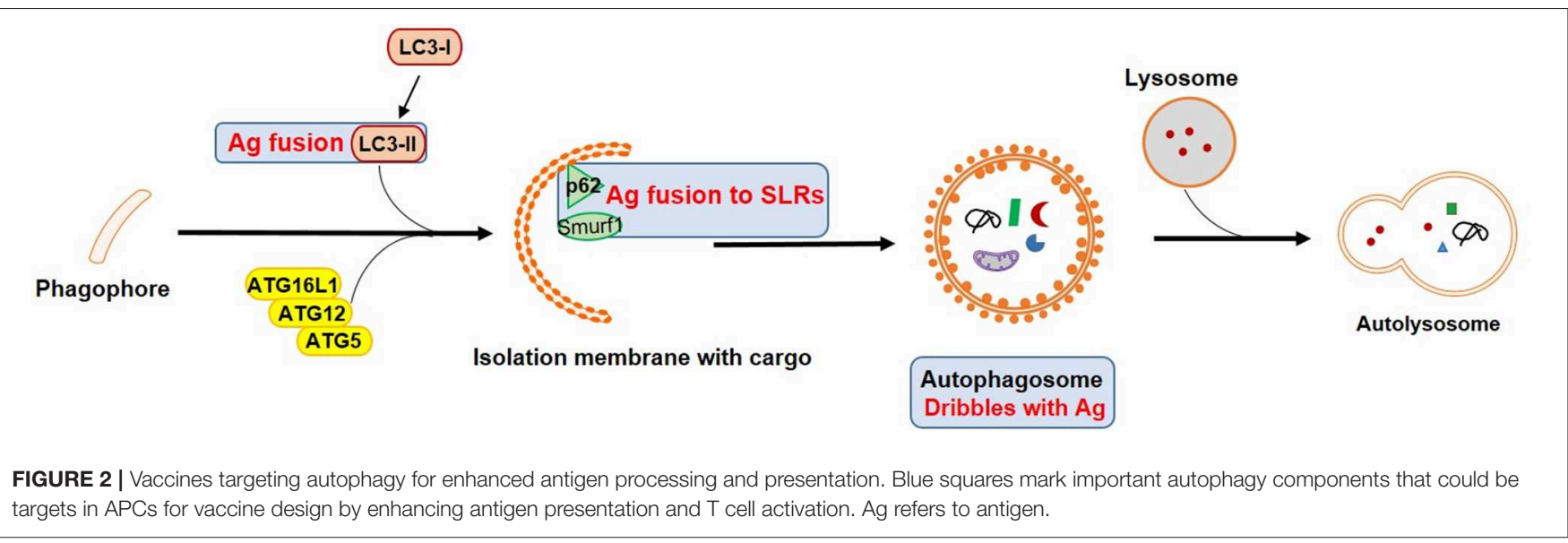


autolysosomes for clearance $(32,33)$, while clearance of Bacillus anthracis is based on rapid induction of LC3 conversion, Beclin1 expression and p62-mediated degradation in neutrophils (34). Redirection of vaccine antigens from proteasomal degradation into autophagosomal pathways could increase the generation and variability of antigen-specific T cells. Fusion of HIV-1 Gagp24 to the selective autophagy receptor sequestosome 1 (SQSTM1)/p62 complex enhanced antigen delivery and increased antigenspecific T cell responses in comparison to Gagp24 alone (35). The connection of p62 and autophagy is highly conserved between species and could be an interesting candidate for T-cell-based vaccine strategies in humans. More recently, another recognition molecule in selective autophagy captured attention regarding autophagy-mediated host defense against infection. Smurf1 is an E3 ubiquitin ligase and a key component in autophagic targeting of $\mathrm{Mtb}$ in macrophages supporting host defense in vivo (36) which may suggest a new potential target for enhancing xenophagic degradation.

Recently, a self-assembling peptide vaccine in which the amphipathic peptide KFE8 (FKFEFKFE) was either combined with MHC class II restricted epitopes from Mtb Ag85B or MHC class I restricted peptides from ovalbumin. These conjugate vaccines were tested in vitro in APCs with known ability to induce strong antibody and cellular responses to conjugated antigens. Interestingly, both variations were processed through autophagy and displayed a highly efficient antigen presentation capacity to $\mathrm{T}$ cells (37). However, the vaccine efficacy still needs to be established in vivo and for other target antigens.

\section{ADJUVANTS THAT ENHANCE VACCINE EFFICIENCY THROUGH AUTOPHAGY}

Some vaccines are derived from attenuated strains of pathogens. Deleting virulence genes increases the vaccine safety but sometimes also reduces immunogenicity, especially when the lost genes are associated with autophagy functions. In order to enhance vaccine efficacy, the boosting of host immune responses with adjuvants which induce autophagy may increase phagocytosis and clearance of pathogens as well as antigen presentation by innate immune cells (Figure 3).

For instance, Bacillus Calmette-Guerin (BCG) representing a live attenuated strain from Mycobacterium tuberculosis (Mtb) is still used as a vaccine for tuberculosis (TB). However, it's efficiency varies and is especially low in adults. Therefore, vaccine adjuvants have gained great interest to improve BCG vaccines. Compared to $\mathrm{Mtb}$, the attenuated BCG lacks a functional ESX1 system (secreting ESAT-6 and CFP-10). This system allows cytosolic components of ubiquitin-mediated autophagy to access phagosomes and to free contained mycobacteria which supports bacterial evasion from xenophagic elimination (38) and reduces antigen presentation (39). Combination of BCG vaccines with autophagy inducers or with peptides from the above mentioned

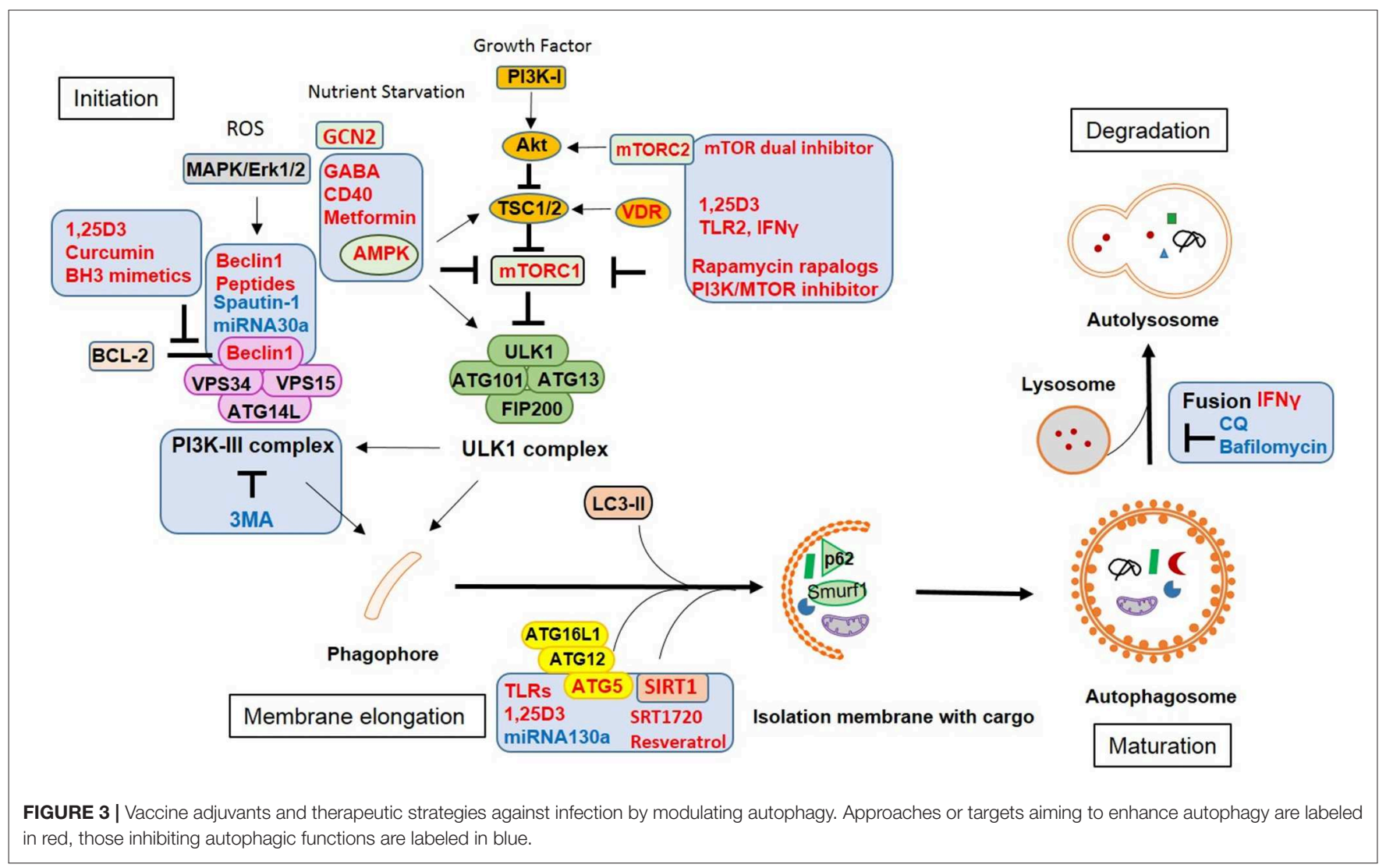


virulence proteins showed better protection in vivo than BCG alone. A BCG vaccine that overexpressed immunogenic Ag85B has been proven superior compared to the wild-type BCG vaccine. Particularly, additional application of rapamycin enhanced Ag85B-specific MHC class II presentation by DCs via autophagy and thus increased vaccine efficacy (40). An autophagy inducing and TLR2 activating C5 peptide from Mtb-derived CFP-10 protein was overexpressed in BCG in combination with $\mathrm{Ag} 85 \mathrm{~B}\left(\mathrm{BCG}^{85 \mathrm{C} 5}\right)$. This recombinant $\mathrm{BCG}^{85 \mathrm{C} 5}$ induced robust LC3-dependent autophagy in macrophages which increased antigen presentation to $\mathrm{CD} 4+\mathrm{T}$ cells in vitro and enhanced effector and central memory $\mathrm{T}$ cell responses in vivo (39). Accordingly, a recombinant BCG $\Delta$ ureC::hly $(+)$ (rBCG) vaccine with enhanced AIM2 inflammasome activation and autophagy was more efficient against TB in preclinical animal models than parental BCG (41). Consequently, triggering other synergistic innate pathways in conjunction with autophagy should boost the immune response and vaccine efficacy.

DCs play a key role for antigen presentation and $\mathrm{T}$ cell induction during viral infections. Autophagy has been shown to be involved in DC maturation during RSV infection (42) which enabled DCs to migrate to secondary lymphoid tissues, interact with naïve T cells and trigger effector T cell responses (43). HIV1 protein Env has been proposed to downregulate autophagy by activating mTOR and reducing lysosomal enzyme activity in DCs, thereby inhibiting antigen presentation and enhancing the transfer of infection into CD4+ T cells $(22,44)$. However, viral inhibition was bypassed by rapamycin-mediated stimulation of autophagy in DCs and restored CD4+ T cell responses (44). Starvation is an efficient way to induce autophagy. By activating the cellular starvation sensor general control non-derepressible 2 (GCN2) kinase, yellow fever vaccine YF-17D induced autophagy in human DCs which operated via the induction of cell death in infected "donor" cells and the enhanced uptake by bystander DCs through LAP, which subsequently resulted in enhanced antigen presentation and robust $\mathrm{T}$ cell responses (45). Beclin1, one important component of canonical autophagy also proved to act as a potent vaccine adjuvant. By enhancing autophagy-mediated antigen presentation, a combinatory HPV-16 E7/Beclin1 DNA vaccine led to superior lymphocyte proliferation and cytotoxicity as compared to the HPV-16 E7 DNA vaccine alone (46).

Additional to traditional adjuvants inducing autophagy such as rapamycin, several drugs may be repurposed from their original usage (e.g., against cancer or diabetes), since they activate autophagy and increase vaccine-specific immune responses. For instance, curcumin is known for inducing autophagy in tumor cells and is currently tested for tumor therapy. Due to its poor intestinal absorption and rapid metabolism, this drug has clinical limitations. However, a recent paper demonstrated that a nanoparticle-formulated version of curcumin could overcome these limitations and optimized various APC functions including autophagy and finally enhanced BCG vaccine efficacy by inducing central memory $\mathrm{T}$ cell as well as Th1 and Th17 responses (47). Another biological, the antioxidant glutathione (GSH), improved BCG vaccination by increasing autophagy and the production of IFN $\gamma$ and $\mathrm{TNF} \alpha$ which prevented loss of $\mathrm{T}$ cells by decreasing the expression of PD-1 (48). However, those adjuvants have not been widely used in other infection models and the effect could be pathogen-specific.

\section{THERAPEUTIC APPROACHES AGAINST INFECTIONS BY MODULATING AUTOPHAGY}

Since autophagy has important functions in innate and adaptive immunity, it is not surprising that pathogens employ autophagy to escape the host immune response. In macrophages, the Grampositive bacteria Listeria monocytogenes (Lm), mycobacteria (Mtb) including BCG, and parasites such as Toxoplasma gondii ( $T$. gondii) are well-studied intracellular pathogens that have different strategies to utilize autophagy for longterm survival or replication (49), while LAP (5) as well as xenophagy (11) usually result in bacterial degradation by lysosome fusion. However, Lm masks its surface with ActA and InlK to avoid recognition (50, 51), while Mtb evades killing by CpsA which prevents a robust oxidative response (ROS) and further leads to LAP (52). To bypass these defects, treatment of innate immune cells with autophagy inducers could enhance pathogen killing (Figure 3). Common therapies include adenosine monophosphate-activated protein kinase (AMPK) modulators. CD40 may stimulate autophagic killing of $T$. gondii in macrophages via calcium/calmodulindependent kinase kinase $ß$ (CaMKKß), AMPK and ULK1 (53) and subsequent fusion of vacuoles and late lysosomes (54). Gamma-aminobutyric acid (GABA) or GABAergic drugs promote autophagy via intracellular calcium release and AMPK signaling resulting in enhanced phagosomal maturation and subsequently antimicrobial responses against mycobacterial infection (55). The U.S. Food and Drug Administration (FDA)approved antidiabetic drug metformin increases the production of mitochondrial reactive oxygen species (ROS) and facilitates phagosome-lysosome fusion which has been shown to limit Mtb growth and to reduce chronic inflammation in infected mice (56).

Another option to enhance autophagy is through vitamin D receptor (VDR) activation and cathelicidin induction. The active form of vitamin D, 1,25-dihydroxyvitamin D3 (1,25D3), induces autophagy in human monocytes and activates transcription of the autophagy-related genes Beclin1 and ATG5 (57). A combination of retinoic acid (RA) and vitamin D3 (VD) (RAVD) enhanced the levels of DC-SIGN and mannose receptors on THP-1 macrophages which increased mycobacterial uptake and inhibited the subsequent intracellular growth of Mtb by inducing ROS and autophagy (58). TLR2/1/CD14 stimulation activates antibacterial autophagy through VDR and cathelicidin in human primary monocytes (59). Additionally, some effector cytokines such as IFN $\gamma$ activate autophagy in macrophages. IFN $\gamma$ treated macrophages could overcome the inhibition of phagosome-lysosome fusion by Mtb and controlled intracellular Mtb growth (60). In severely ill TB patients, IFN $\gamma$ as well as Th1 and Th17 immune responses are required to eliminate Mtb. Interestingly, IL17a alone was unable to augment autophagy because of a disease-associated defect in MAPK1/3 signaling. Adding IFN $\gamma$ to IL17a increased autophagy levels in the patients' 
monocytes resulting in strong immunity to Mtb and promoting mycobacterial killing (61).

Though autophagy plays a key role in macrophages and neutrophils for pathogen clearance through xenophagy or LAP, it is still unclear how phagosomes trigger autophagy. One paper suggests a connection to TLR signaling, but this has not been investigated in infectious context (62). Autophagy may not always work best to eliminate pathogens. Previous reports have shown that phagocytosis of Escherichia coli triggers the autophagic machinery in neutrophils (63). On the other hand, autophagy may reduce the phagocytosis rate of mycobacteria in murine macrophages (64) and neutrophils (65). The reduced internalization is due to decreased expression of two class A scavenger receptors, namely macrophage receptor with collagenous structure (MARCO) and macrophage scavenger receptor 1 (MSR1) (66). Therefore, pharmacological modulation of autophagy should aim to target both, autophagy and phagocytosis, and should be carefully designed for each pathogen.

Clearing blood-borne pathogens is a hallmark feature of the spleen. In this secondary lymphoid organ, pDCs and red pulp macrophages efficiently cleared malaria parasites after boosting autophagy with rapamycin $(67,68)$. The treatment enhanced antigen presentation in these cells and shifted the cytokine and chemokines profile in vivo which recruited effector cells into the spleen and enhanced $\mathrm{T}$ cell responses. Thus, targeting autophagy in $\mathrm{pDCs}$ and red pulp macrophages may open new prospects for the development of novel antimalarial drugs.

When it comes to viral infection, some viral proteins directly inhibit autophagy. For instance, HIV-1 Nef is interacting with Beclin1 which blocks the late stage of autophagy in macrophages, thereby protecting virus particles from degradation (69). That is able to suppress IFN $\gamma$-induced autophagy in infected macrophages (70) as well as in bystander macrophages through Src-Akt and STAT3 signaling (71). Rescue of autophagy function could be a novel approach to prevent and treat HIV-1 infection and related opportunistic infections. The PI3K/MTOR inhibitor (dactolisib) and PI3K/MTOR/BRD4 inhibitor (SF2523, JQ1) restrain HIV replication through degradative autophagy without altering the initial susceptibility of macrophages to infection (72). Low vitamin D levels in HIV-1 infected patients are associated with more rapid disease progression and increased risk for other infections such as Mtb. 1,25D3 targets multiple steps in autophagy and inhibits HIV replication and mycobacterial growth in co-infected human macrophages through the induction of autophagy (73). In RSV infection, Sirtuin 1 (SIRT1) an NAD+ dependent deacetylase regulates autophagy depending on the nutrient status. SIRT1 may induce autophagy directly by deacetylating TAG5 and 7 and LC3. Activated DCs produce crucial cytokines promoting antiviral Th1 responses, while pathologic Th2 and Th17 responses are suppressed during infection (74). SIRT1 inducers like SRT1720 could be a therapeutic alternative for RSV patients.

Autophagy may have distinct functions depending on the stage of infection or the host cell type infected $(75,76)$. In the early phase of HSV-1 infection, autophagy was found to be transiently induced in human THP-1 cells favoring viral replication (77). In the later phase, however, viral protein ICP34.5 blocked the maturation of autophagosomes which reduced viral antigen presentation by DCs (17). Alternatively, human gamma herpesviruses EBV and KSHV regulate autophagy in immune cells during de novo infection, while autophagy plays a distinct role in chronic murine gamma herpesvirus 68 (MHV68) infection by triggering virus reactivation from latency (78). Thus, opposing effects of the same drug may occur depending on the infection settings. While rapamycin induces killing of Mtb in macrophages, it supports Mtb growth during lowdose and controlled infection when co-infected with HIV-1 by interfering with phagosomal maturation (35). Therefore, a detailed knowledge of how pathogens modulate autophagy during the infection cycle will help to develop more specific targets for autophagy-based strategies against infectious diseases.

Various viral virulence factors such as ICP34.5 (HSV-1), Nef (HIV-1), or M11 (MHV-68) have been shown to specifically interact with autophagy-related proteins such as Beclin1 to exploit autophagy for viral replication. New therapy approaches that target specific components of autophagy pathways, which are manipulated by pathogens, should maximize clinical benefits while minimizing toxicity. For instance, an autophagy inducing cell-permeable peptide (Tat-Beclin1) has been generated by mapping the functional region of Beclin1 with the HIV-1 Tat transduction domain (PTD). Therapeutic application of Tat-Beclin1 was associated with reduced HIV-1 replication, decreased intracellular survival of the bacterium $\mathrm{Lm}$ in human macrophages and reduced mortality of mice infected with chikungunya or West Nile virus (79). Inhibitors of interactions between viral and autophagic proteins may also have potential benefits for the prevention and treatment of a broad range of human diseases. Bcl-2 binds to Beclin1 preventing assembly of pre-autophagosomal structures which inhibits autophagy. This interaction involves a Bcl-2 homology 3 (BH3) domain in Beclin1. Proteins containing $\mathrm{BH} 3$ domains such as $\mathrm{BH} 3$ mimetics $(80,81)$ can competitively disrupt the interaction between Beclin1 and Bcl-2 and thereby induce autophagy (82). Another example is MHV-68 M11 which binds to one BH3 domain of Beclin1 and inhibits autophagy. Alternatively, a Beclin1 BH3 domain-derived peptide which selectively binds to M11, but not to Bcl-2, abrogated M11-mediated down-regulation of autophagy (83) (Figure 3).

Autophagy is additionally regulated by mitochondrial integrity and contributes to the elimination of damaged organelles. Autophagy may selectively degrade inflammasome components such as NLRP3 and products such as Pro-IL1ß (84) thereby limiting the secretion of pro-inflammatory cytokines. As a result, the dampening of inflammation is another important role of autophagy in innate immune cells which so far has been exclusively shown in myeloid cells. For instance, loss of autophagy-related proteins in macrophages causes massive inflammation after infection which may lead to host tissue damage such as lung injury in $\operatorname{IAV}(85,86)$ and Mtb infection (87) as well as in pseudomonas aeruginosa-driven abdominal infection (88). Autophagy also helps to curtail virus-induced systemic inflammation by creating an environment that prevents host injury mediated by pro-inflammatory cytokines. 
Trichostatin A (TSA), an autophagy inducer used to reduce systemic inflammation and to attenuate sepsis-induced organ injury, promotes M2 polarization in peritoneal macrophages and ultimately improved the survival of mice with polymicrobial sepsis (89). In some autoimmune diseases such as inflammatory bowel disease (IBD), the containment of inflammation may prevent the development of disease and reduce the risk of infections. Enhancing autophagy may be therapeutically beneficial by regulating inflammation and clearing intestinal pathogens. Recently, IL-10 was reported to induce mitophagy (autophagy-mediated mitochondria degradation) to prevent accumulation of dysfunctional mitochondria and production of mitochondrial ROS in macrophages from IBD patients (90) (Figure 3).

\section{THERAPEUTIC STRATEGIES AIMING TO INHIBIT AUTOPHAGY TO BLOCK VIRUS REPLICATION AND IMMUNE EVASION}

Autophagy seems to be an angel for most infection scenarios, except for two: first, autophagy may benefit virus replication rather than support host immune protection as seen for flaviviruses. Second, autophagy may degrade components of innate immune pathways which negatively affects innate sensing of pathogens and antiviral cytokine production. The former represents a potential therapeutic target for several (re-)emerging diseases for which we currently have no vaccine available due to rapid spread and high virulence. The latter is exemplified by multiple targets in the cGAS/STING DNA sensing pathway aiming to halt IFN production. A direct interaction between cGAS and Beclin1 has been described in macrophages during HSV-1 infection (91). Similarly, p62mediated degradation of cGAS in HSV-1 and VSV infection (92) as well as poxin-mediated cGAMP degradation by various poxviruses has been reported (93). The latter has not been associated with autophagy so far, but the therapeutic potential of any autophagy modulator that may arise from these studies has not been assed and molecular mechanisms still need to be elucidated.

Some viruses induce autophagy for their own replication by taking advantage of membrane structures produced in this process as reported for flaviviruses [hepatitis $\mathrm{C}$ virus (HCV), dengue virus (DENV), zika virus (ZIKV)] or hepatitis $B$ virus (HBV) (94). For viruses that infect specific organs or tissues like $\mathrm{HBV}$ or $\mathrm{HCV}$, tissue-specific targeting of autophagy e.g., hepatocytes, would be favorable. HCV genomic RNA is recognized by RIG-I, MDA5 and TLRs to activate IFN signaling and pro-inflammatory cytokine secretion. HCV induces autophagy in hepatocytes which enables its replication and trafficking, additionally, attenuates the innate immune response by viral proteins NS3 and NS5A (95). Consequently, knockdown of autophagy-related proteins (Beclin1 or ATG7) in immortalized human hepatocytes (IHH) could inhibit HCV growth (96) and block exosome-mediated virus transmission (97).

For DENV and ZIKV, some autophagy pathway components are crucial for replication including maturation and packaging.
The induction of autophagy by ZIKV appears to be linked to the activation of AMPK, while DENV induces autophagy by activation of VPS34 (98). USP10 and USP13 are needed to reverse ubiquitination and subsequently degradation of the Beclin1-VPS34-ATG14 complex. Targeting this complex by Spautin1 which inhibits the deubiquitination activity of the two molecules inhibited DENV infection (99). In innate immune cells, the pro-inflammatory cytokine macrophage migration inhibitory factor (MIF) induces autophagy and facilitates DENV replication. Inhibition of MIF-induced autophagy by minocycline might represent an alternative therapeutic approach against DENV infection (100). Pharmacological inhibitors of autophagolysosomal activity such as chloroquine (CQ) prevents endosomal viral RNA release and autophagy-dependent viral replication and is currently used to prevent maternal to fetal transmission of ZIKV (101-103) (Figure 3).

MicroRNAs (miRNAs) represent a new tool to regulate autophagy by specifically targeting the expression of autophagyrelated genes. They bind to the target mRNA through specific base-pairing interactions between the "seed" region of miRNA and sites within coding and untranslated regions (UTRs) of mRNAs, especially $3^{\prime}$ UTRs, to suppress gene expression. Several miRNAs have been shown to augment or repress virus replication through interfering with autophagy. Therefore, manipulation of cellular miRNAs which target autophagy components represent a novel approach for purging pathogens. Administration of miRNA130a diminished HCV replication by interfering with ATG5-dependent autophagy (104), while miRNA146a targets TRAF6 which blocked DENV-induced autophagy in THP-1 cells (105). Enterovirus 71 (EV71)-induced autophagy is mediated by Beclin 1 which contains a potential binding site for miRNA30a. By using a miRNA30a mimic, EV71 replication was suppressed by blocking virus-induced autophagy (106). This therapeutic approach is widely used in the tumor field, but the options for different pathogens may vary, since miRNA expression is altered during conditions of stress and disease (Figure 3).

\section{FUTURE PROSPECTS}

Innate immune cells are the first line of defense against infection. Targeting autophagy in those cells is an attractive approach to augment vaccination efficacy or to improve immunotherapeutic strategies against infectious diseases. Vaccines which are based on genetic fusion of antigens to important components of autophagy pathways improved adaptive immune responses by enhancing antigen processing and presentation in APCs. Combination with autophagy modulators as adjuvants has been proven to further boost host immune responses by triggering innate immunity as well as increasing immune cell functions such as cytokine/chemokine production, maturation, and migration. Pharmacological induction of autophagy could increase pathogen clearance in phagocytes through xenophagy or LAP. Furthermore, given the potent anti-inflammatory effect associated with autophagy, employing autophagic functions in myeloid cells might also help to control infections in some 
autoimmune diseases. The functions or effector mechanisms exerted by or related to autophagy during infection may vary among cell types, the type of pathogen or the stage of infection. The impact of autophagy goes beyond single cell types and involves intensive cross-talk within the whole immune system and therapeutic strategies may have to be determined individually for a given pathogen. Future studies will have to focus on investigating the role of autophagy for pathogenhost-specific interplay in vivo and identify relevant steps in the course of infection in which the targeting of autophagypossibly in selected cell types-proves to be most efficient for pathogen clearance and protection. This will allow to develop new strategies for vaccines or therapeutic approaches

\section{REFERENCES}

1. Kuballa P, Nolte WM, Castoreno AB, Xavier RJ. Autophagy and the immune system. Annu Rev Immunol. (2012) 30:611-46. doi: 10.1146/annurev-immunol-020711-074948

2. Ma Y, Galluzzi L, Zitvogel L, Kroemer G. Autophagy and cellular immune responses. Immunity. (2013) 39:211-27. doi: 10.1016/j.immuni.2013.07.017

3. Choi Y, Bowman JW, Jung JU. Autophagy during viral infection - a double-edged sword. Nat Rev Microbiol. (2018) 16:341-54. doi: 10.1038/s41579-018-0003-6

4. Codogno P, Mehrpour M, Proikas-Cezanne T. Canonical and non-canonical autophagy: variations on a common theme of self-eating? Nat Rev Mol Cell Biol. (2011) 13:7-12. doi: 10.1038/nrm3249

5. Munz C. Non-canonical functions of macroautophagy proteins during endocytosis by myeloid antigen presenting cells. Front Immunol. (2018) 9:2765. doi: 10.3389/fimmu.2018.02765

6. Gui X, Yang H, Li T, Tan X, Shi P, Li M, et al. Autophagy induction via STING trafficking is a primordial function of the cGAS pathway. Nature. (2019) 567:262-6. doi: 10.1038/s41586-019-1006-9

7. Ghislat G, Lawrence T. Autophagy in dendritic cells. Cell Mol Immunol. (2018) 15:944-52. doi: $10.1038 / \mathrm{cmi} .2018 .2$

8. Munz C. Autophagy beyond intracellular MHC class II antigen presentation. Trends Immunol. (2016) 37:755-63. doi: 10.1016/j.it.2016.08.017

9. Merkley SD, Chock CJ, Yang XO, Harris J, Castillo EF. Modulating T cell responses via autophagy: the intrinsic influence controlling the function of both antigen-presenting cells and T Cells. Front Immunol. (2018) 9:2914. doi: 10.3389/fimmu.2018.02914

10. Romao S, Munz C. LC3-associated phagocytosis. Autophagy. (2014) 10:5268. doi: 10.4161/auto.27606

11. Sharma V, Verma S, Seranova E, Sarkar S, Kumar D. Selective autophagy and xenophagy in infection and disease. Front Cell Dev Biol. (2018) 6:147. doi: 10.3389/fcell.2018.00147

12. Blum JS, Wearsch PA, Cresswell P. Pathways of antigen processing. Annu Rev Immunol. (2013) 31:443-73. doi: 10.1146/annurev-immunol-032712095910

13. Thiele F, Tao S, Zhang Y, Muschaweckh A, Zollmann T, Protzer $\mathrm{U}$, et al. Modified vaccinia virus Ankara-infected dendritic cells present $\mathrm{CD}^{+}$T-cell epitopes by endogenous major histocompatibility complex class II presentation pathways. J Virol. (2015) 89:2698-709. doi: 10.1128/JVI.03244-14

14. Lee HK, Mattei LM, Steinberg BE, Alberts P, Lee YH, Chervonsky A, et al. In vivo requirement for Atg5 in antigen presentation by dendritic cells. Immunity. (2010) 32:227-39. doi: 10.1016/j.immuni.2009.12.006

15. Gobeil PA, Leib DA. Herpes simplex virus gamma34.5 interferes with autophagosome maturation and antigen presentation in dendritic cells. MBio. (2012) 3:e00267-12. doi: 10.1128/mBio.00267-12

16. Jiang Y, Yin X, Stuart PM, Leib DA. Dendritic cell autophagy contributes to herpes simplex virus-driven stromal keratitis and immunopathology. MBio. (2015) 6:e01426-15. doi: 10.1128/mBio.01426-15 with optimized efficacy against infectious diseases and help to minimize unwanted off-target effects or toxicity.

\section{AUTHOR CONTRIBUTIONS}

ST and ID wrote the manuscript. ST generated images.

\section{FUNDING}

This work was supported by research funding from the Deutsche Forschungsgemeinschaft (DFG) grant 1949/1 to ID, the European Union grant 812915 VacPath to ID, and the MOI III Graduate School.

17. Budida R, Stankov MV, Dohner K, Buch A, Panayotova-Dimitrova D, Tappe $\mathrm{KA}$, et al. Herpes simplex virus 1 interferes with autophagy of murine dendritic cells and impairs their ability to stimulate CD8(+) T lymphocytes. Eur J Immunol. (2017) 47:1819-34. doi: 10.1002/eji.201646908

18. Liu E, Van Grol J, Subauste CS. Atg 5 but not Atg7 in dendritic cells enhances IL-2 and IFN-gamma production by Toxoplasma gondii-reactive CD4+ T cells. Microbes Infect. (2015) 17:275-84. doi: 10.1016/j.micinf.2014.12.008

19. Reed M, Morris SH, Jang S, Mukherjee S, Yue Z, Lukacs NW. Autophagyinducing protein beclin-1 in dendritic cells regulates CD4 $\mathrm{T}$ cell responses and disease severity during respiratory syncytial virus infection. J Immunol. (2013) 191:2526-37. doi: 10.4049/jimmunol.1300477

20. Jin Y, Sun C, Feng L, Li P, Xiao L, Ren Y, et al. Regulation of SIV antigen-specific CD4+ T cellular immunity via autophagosome-mediated MHC II molecule-targeting antigen presentation in mice. PLoS ONE. (2014) 9:e93143. doi: 10.1371/journal.pone.0093143

21. Coulon PG, Richetta C, Rouers A, Blanchet FP, Urrutia A, Guerbois M, et al. HIV-infected dendritic cells present endogenous MHC class II-restricted antigens to HIV-specific CD4+ T cells. J Immunol. (2016) 197:517-32. doi: 10.4049/jimmunol.1600286

22. Harman AN, Kraus M, Bye CR, Byth K, Turville SG, Tang O, et al. HIV1 -infected dendritic cells show 2 phases of gene expression changes, with lysosomal enzyme activity decreased during the second phase. Blood. (2009) 114:85-94. doi: 10.1182/blood-2008-12-194845

23. Schmid D, Pypaert M, Munz C. Antigen-loading compartments for major histocompatibility complex class II molecules continuously receive input from autophagosomes. Immunity. (2007) 26:79-92. doi: 10.1016/j.immuni.2006.10.018

24. Zhao F, Zhai Y, Zhu J, Xiao P, Feng G. Enhancement of autophagy as a strategy for development of new DNA vaccine candidates against Japanese encephalitis. Vaccine. (2019) 37:5588-95. doi: 10.1016/j.vaccine.2019.07.093

25. Villadangos JA, Young L. Antigen-presentation properties of plasmacytoid dendritic cells. Immunity. (2008) 29:352-61. doi: 10.1016/j.immuni.2008.09.002

26. Sheng J, Chen Q, Soncin I, Ng SL, Karjalainen K, Ruedl C. A Discrete subset of monocyte-derived cells among typical conventional type 2 dendritic cells can efficiently cross-present. Cell Rep. (2017) 21:1203-14. doi: 10.1016/j.celrep.2017.10.024

27. English L, Chemali M, Duron J, Rondeau C, Laplante A, Gingras D, et al. Autophagy enhances the presentation of endogenous viral antigens on $\mathrm{MHC}$ class I molecules during HSV-1 infection. Nat Immunol. (2009) 10:480-7. doi: 10.1038/ni.1720

28. Tey SK, Khanna R. Autophagy mediates transporter associated with antigen processing-independent presentation of viral epitopes through MHC class I pathway. Blood. (2012) 120:994-1004. doi: 10.1182/blood-2012-01-402404

29. Zang F, Chen Y, Lin Z, Cai Z, Yu L, Xu F, et al. Autophagy is involved in regulating the immune response of dendritic cells to influenza $A$ (H1N1) pdm09 infection. Immunology. (2016) 148:56-69. doi: 10.1111/imm.12587

30. Ye W, Xing Y, Paustian C, Van De Ven R, Moudgil T, Hilton TL, et al. Cross-presentation of viral antigens in dribbles leads to efficient activation 
of virus-specific human memory T cells. J Transl Med. (2014) 12:100. doi: 10.1186/1479-5876-12-100

31. Fan J, Wu Y, Jiang $M$, Wang $L$, Yin $D$, Zhang $Y$, et al. IFN-DC loaded with autophagosomes containing virus antigen is highly efficient in inducing virus-specific human T cells. Int J Med Sci. (2019) 16:741-50. doi: $10.7150 /$ ijms.31830

32. Ponpuak M, Davis AS, Roberts EA, Delgado MA, Dinkins C, Zhao Z, et al. Delivery of cytosolic components by autophagic adaptor protein p62 endows autophagosomes with unique antimicrobial properties. Immunity. (2010) 32:329-41. doi: 10.1016/j.immuni.2010.02.009

33. Kimmey JM, Huynh JP, Weiss LA, Park S, Kambal A, Debnath J, et al. Unique role for ATG5 in neutrophil-mediated immunopathology during $M$. tuberculosis infection. Nature. (2015) 528:565-9. doi: 10.1038/nature16451

34. Ramachandran G, Gade P, Tsai P, Lu W, Kalvakolanu DV, Rosen GM, et al. Potential role of autophagy in the bactericidal activity of human PMNs for Bacillus anthracis. Pathog Dis. (2015) 73:ftv080. doi: 10.1093/femspd/ftv080

35. Andersson AM, Andersson B, Lorell C, Raffetseder J, Larsson M, Blomgran R. Autophagy induction targeting mTORC1 enhances Mycobacterium tuberculosis replication in HIV co-infected human macrophages. Sci Rep. (2016) 6:28171. doi: 10.1038/srep28171

36. Franco LH, Nair VR, Scharn CR, Xavier RJ, Torrealba JR, Shiloh MU, et al. The ubiquitin ligase smurf1 functions in selective autophagy of Mycobacterium tuberculosis and anti-tuberculous host defense. Cell Host Microbe. (2017) 21:59-72. doi: 10.1016/j.chom.2016.11.002

37. Rudra JS, Khan A, Clover TM, Endsley JJ, Zloza A, Wang J, et al. Supramolecular peptide nanofibers engage mechanisms of autophagy in antigen-presenting cells. ACS Omega. (2017) 2:9136-43. doi: 10.1021/acsomega.7b00525

38. Watson RO, Manzanillo PS, Cox JS. Extracellular M. tuberculosis DNA targets bacteria for autophagy by activating the host DNA-sensing pathway. Cell. (2012) 150:803-15. doi: 10.1016/j.cell.2012.06.040

39. Khan A, Bakhru P, Saikolappan S, Das K, Soudani E, Singh CR, et al. An autophagy-inducing and TLR-2 activating BCG vaccine induces a robust protection against tuberculosis in mice. NPJ Vaccines. (2019) 4:34. doi: 10.1038/s41541-019-0122-8

40. Jagannath C, Lindsey DR, Dhandayuthapani S, Xu Y, Hunter RL Jr, Eissa NT. Autophagy enhances the efficacy of BCG vaccine by increasing peptide presentation in mouse dendritic cells. Nat Med. (2009) 15:267-76. doi: $10.1038 / \mathrm{nm} .1928$

41. Saiga H, Nieuwenhuizen N, Gengenbacher M, Koehler AB, Schuerer S, Moura-Alves $\mathrm{P}$, et al. The Recombinant BCG DeltaureC::hly vaccine targets the AIM2 inflammasome to induce autophagy and inflammation. J Infect Dis. (2015) 211:1831-41. doi: 10.1093/infdis/jiu675

42. Morris S, Swanson MS, Lieberman A, Reed M, Yue Z, Lindell DM, et al. Autophagy-mediated dendritic cell activation is essential for innate cytokine production and APC function with respiratory syncytial virus responses. $J$ Immunol. (2011) 187:3953-61. doi: 10.4049/jimmunol.1100524

43. Dalod M, Chelbi R, Malissen B, Lawrence T. Dendritic cell maturation: functional specialization through signaling specificity and transcriptional programming. EMBO J. (2014) 33:1104-16. doi: 10.1002/embj.201488027

44. Blanchet FP, Moris A, Nikolic DS, Lehmann M, Cardinaud S, Stalder R, et al. Human immunodeficiency virus-1 inhibition of immunoamphisomes in dendritic cells impairs early innate and adaptive immune responses. Immunity. (2010) 32:654-69. doi: 10.1016/j.immuni.2010.04.011

45. Ravindran R, Khan N, Nakaya HI, Li S, Loebbermann J, Maddur MS, et al. Vaccine activation of the nutrient sensor GCN2 in dendritic cells enhances antigen presentation. Science. (2014) 343:313-7. doi: $10.1126 /$ science. 1246829

46. Naziri H, Tahamtan A, Dadmanesh M, Barati M, Ghorban K. Antitumor effects of HPV DNA vaccine adjuvanted with beclin-1 as an autophagy inducer in a mice model. Iran Biomed J. (2019) 23:388-94. doi: $10.29252 /$ ibj.23.6.388

47. Ahmad S, Bhattacharya D, Kar S, Ranganathan A, Van Kaer L, Das G. Curcumin nanoparticles enhance Mycobacterium bovis BCG vaccine efficacy by modulating host immune responses. Infect Immun. (2019) 87:e0291-19. doi: 10.1128/IAI.00291-19

48. Abrahem R, Cao R, Robinson B, Munjal S, Cho T, To K, et al. Elucidating the efficacy of the bacille calmette-guerin vaccination in conjunction with first line antibiotics and liposomal glutathione. J Clin Med. (2019) 8:1556. doi: $10.3390 / \mathrm{jcm} 8101556$

49. Mitchell G, Chen C, Portnoy DA. Strategies Used by Bacteria to Grow in Macrophages. Microbiol Spectr. (2016) 4:MCHD-0012-2015. doi: 10.1128/microbiolspec.MCHD-0012-2015

50. Mitchell G, Ge L, Huang Q, Chen C, Kianian S, Roberts MF, et al. Avoidance of autophagy mediated by PlcA or ActA is required for Listeria monocytogenes growth in macrophages. Infect Immun. (2015) 83:2175-84. doi: 10.1128/IAI.00110-15

51. Mitchell G, Cheng MI, Chen C, Nguyen BN, Whiteley AT, Kianian S, et al. Listeria monocytogenes triggers noncanonical autophagy upon phagocytosis, but avoids subsequent growth-restricting xenophagy. Proc Natl Acad Sci USA. (2018) 115:E210-7. doi: 10.1073/pnas.1716055115

52. Koster S, Upadhyay S, Chandra P, Papavinasasundaram K, Yang G, Hassan A, et al. Mycobacterium tuberculosis is protected from NADPH oxidase and LC3-associated phagocytosis by the LCP protein CpsA. Proc Natl Acad Sci USA. (2017) 114:E8711-20. doi: 10.1073/pnas.170 7792114

53. Liu E, Lopez Corcino Y, Portillo JA, Miao Y, Subauste CS. Identification of signaling pathways by which CD40 stimulates autophagy and antimicrobial activity against Toxoplasma gondii in macrophages. Infect Immun. (2016) 84:2616-26. doi: 10.1128/IAI.00101-16

54. Andrade RM, Wessendarp M, Gubbels MJ, Striepen B, Subauste CS. CD40 induces macrophage anti-Toxoplasma gondii activity by triggering autophagy-dependent fusion of pathogen-containing vacuoles and lysosomes. J Clin Invest. (2006) 116:2366-77. doi: 10.1172/JCI28796

55. Kim JK, Kim YS, Lee HM, Jin HS, Neupane C, Kim S, et al. GABAergic signaling linked to autophagy enhances host protection against intracellular bacterial infections. Nat Commun. (2018) 9:4184. doi: 10.1038/s41467-018-06487-5

56. Singhal A, Jie L, Kumar P, Hong GS, Leow MK, Paleja B. et al. Metformin as adjunct antituberculosis therapy. Sci Transl Med. (2014) 6:263ra159. doi: $10.1126 /$ scitranslmed.3009885

57. Yuk JM, Shin DM, Lee HM, Yang CS, Jin HS, Kim KK, et al. Vitamin D3 induces autophagy in human monocytes/macrophages via cathelicidin. Cell Host Microbe. (2009) 6:231-43. doi: 10.1016/j.chom.2009.08.004

58. Estrella JL, Kan-Sutton C, Gong X, Rajagopalan M, Lewis DE, Hunter RL, et al. A Novel in vitro Human Macrophage Model to Study the Persistence of Mycobacterium tuberculosis using Vitamin $\mathrm{D}(3)$ and retinoic acid activated THP-1 macrophages. Front Microbiol. (2011) 2:67. doi: 10.3389/fmicb.2011.00067

59. Shin DM, Yuk JM, Lee HM, Lee SH, Son JW, Harding CV, et al. Mycobacterial lipoprotein activates autophagy via TLR2/1/CD14 and a functional vitamin D receptor signalling. Cell Microbiol. (2010) 12:1648-65. doi: 10.1111/j.1462-5822.2010.01497.x

60. Gutierrez MG, Master SS, Singh SB, Taylor GA, Colombo MI, Deretic V. Autophagy is a defense mechanism inhibiting BCG and Mycobacterium tuberculosis survival in infected macrophages. Cell. (2004) 119:753-66. doi: 10.1016/j.cell.2004.11.038

61. Tateosian NL, Pellegrini JM, Amiano NO, Rolandelli A, Casco N, Palmero DJ, et al. IL17A augments autophagy in Mycobacterium tuberculosisinfected monocytes from patients with active tuberculosis in association with the severity of the disease. Autophagy. (2017) 13:1191-204. doi: 10.1080/15548627.2017.1320636

62. Sanjuan MA, Dillon CP, Tait SW, Moshiach S, Dorsey F, Connell S, et al. Toll-like receptor signalling in macrophages links the autophagy pathway to phagocytosis. Nature. (2007) 450:1253-7. doi: 10.1038/nature 06421

63. Mitroulis I, Kourtzelis I, Kambas K, Rafail S, Chrysanthopoulou A, Speletas $\mathrm{M}$, et al. Regulation of the autophagic machinery in human neutrophils. Eur J Immunol. (2010) 40:1461-72. doi: 10.1002/eji.200940025

64. Lima JG, De Freitas Vinhas C, Gomes IN, Azevedo CM, Dos Santos RR, Vannier-Santos MA, et al. Phagocytosis is inhibited by autophagic induction in murine macrophages. Biochem Biophys Res Commun. (2011) 405:604-9. doi: 10.1016/j.bbrc.2011.01.076

65. Jin L, Batra S, Jeyaseelan S. Deletion of Nlrp3 augments survival during polymicrobial sepsis by decreasing autophagy and enhancing phagocytosis. $J$ Immunol. (2017) 198:1253-62. doi: 10.4049/jimmunol.1601745 
66. Bonilla DL, Bhattacharya A, Sha Y, Xu Y, Xiang Q, Kan A, et al. Autophagy regulates phagocytosis by modulating the expression of scavenger receptors. Immunity. (2013) 39:537-47. doi: 10.1016/j.immuni.2013.08.026

67. Sengupta A, Keswani T, Sarkar S, Ghosh S, Mukherjee S, Bhattacharyya A. Autophagic induction modulates splenic plasmacytoid dendritic cell mediated immune response in cerebral malarial infection model. Microbes Infect. (2019) 21:475-84. doi: 10.1016/j.micinf.2019.05.004

68. Sengupta A, Sarkar S, Keswani T, Mukherjee S, Ghosh S, Bhattacharyya A. Impact of autophagic regulation on splenic red pulp macrophages during cerebral malarial infection. Parasitol Int. (2019) 71:18-26. doi: 10.1016/j.parint.2019.03.008

69. Kyei GB, Dinkins C, Davis AS, Roberts E, Singh SB, Dong C, et al. Autophagy pathway intersects with HIV-1 biosynthesis and regulates viral yields in macrophages. J Cell Biol. (2009) 186:255-68. doi: 10.1083/jcb.200 903070

70. Li JC, Au KY, Fang JW, Yim HC, Chow KH, Ho PL, et al. HIV-1 trans-activator protein dysregulates IFN-gamma signaling and contributes to the suppression of autophagy induction. AIDS. (2011) 25:15-25. doi: 10.1097/QAD.0b013e328340fd61

71. Van Grol J, Subauste C, Andrade RM, Fujinaga K, Nelson J, Subauste CS. HIV-1 inhibits autophagy in bystander macrophage/monocytic cells through Src-Akt and STAT3. PLoS ONE. (2010) 5:e11733. doi: 10.1371/journal.pone.0011733

72. Campbell GR, Bruckman RS, Herns SD, Joshi S, Durden DL, Spector SA. Induction of autophagy by PI3K/MTOR and PI3K/MTOR/BRD4 inhibitors suppresses HIV-1 replication. J Biol Chem. (2018) 293:5808-20. doi: 10.1074/jbc.RA118.002353

73. Campbell GR, Spector SA. Vitamin D inhibits human immunodeficiency virus type 1 and Mycobacterium tuberculosis infection in macrophages through the induction of autophagy. PLoS Pathog. (2012) 8:e1002689. doi: 10.1371/journal.ppat.1002689

74. Owczarczyk AB, Schaller MA, Reed M, Rasky AJ, Lombard DB, Lukacs NW. Sirtuin 1 regulates dendritic cell activation and autophagy during respiratory syncytial virus-induced immune responses. J Immunol. (2015) 195:1637-46. doi: 10.4049/jimmunol.1500326

75. Munz C. Autophagy proteins in viral exocytosis and anti-viral immune responses. Viruses. (2017) 9:288. doi: 10.3390/v9100288

76. Ahmad L, Mostowy S, Sancho-Shimizu V. Autophagy-virus interplay: from cell biology to human disease. Front Cell Dev Biol. (2018) 6:155. doi: $10.3389 /$ fcell.2018.00155

77. Siracusano G, Venuti A, Lombardo D, Mastino A, Esclatine A, Sciortino MT. Early activation of MyD88-mediated autophagy sustains HSV-1 replication in human monocytic THP-1 cells. Sci Rep. (2016) 6:31302. doi: $10.1038 /$ srep31302

78. Park S, Buck MD, Desai C, Zhang X, Loginicheva E, Martinez J, et al. Autophagy genes enhance murine gammaherpesvirus 68 reactivation from latency by preventing virus-induced systemic inflammation. Cell Host Microbe. (2016) 19:91-101. doi: 10.1016/j.chom.2015.12.010

79. Shoji-Kawata S, Sumpter R, Leveno M, Campbell GR, Zou Z, Kinch L, et al. Identification of a candidate therapeutic autophagy-inducing peptide. Nature. (2013) 494:201-6. doi: 10.1038/nature11866

80. Maiuri MC, Criollo A, Tasdemir E, Vicencio JM, Tajeddine N, Hickman $\mathrm{JA}$, et al. BH3-only proteins and $\mathrm{BH} 3$ mimetics induce autophagy by competitively disrupting the interaction between Beclin 1 and Bcl-2/BclX(L). Autophagy. (2007) 3:374-6. doi: 10.4161/auto.4237

81. Rubinsztein DC, Codogno P, Levine B. Autophagy modulation as a potential therapeutic target for diverse diseases. Nat Rev Drug Discov. (2012) 11:70930. doi: $10.1038 / \mathrm{nrd} 3802$

82. Levine B, Sinha S, Kroemer G. Bcl-2 family members: dual regulators of apoptosis and autophagy. Autophagy. (2008) 4:600-6. doi: 10.4161/auto.6260

83. Su M, Mei Y, Sanishvili R, Levine B, Colbert CL, Sinha S. Targeting gammaherpesvirus $68 \mathrm{Bcl}-2$-mediated down-regulation of autophagy. J Biol Chem. (2014) 289:8029-40. doi: 10.1074/jbc.M113.515361

84. Harris J, Hartman M, Roche C, Zeng SG, O'shea A, Sharp FA, et al. Autophagy controls IL-1beta secretion by targeting pro-IL-1beta for degradation. J Biol Chem. (2011) 286:9587-97. doi: 10.1074/jbc.M110.202911

85. Lupfer C, Thomas PG, Anand PK, Vogel P, Milasta S, Martinez J, et al. Receptor interacting protein kinase 2-mediated mitophagy regulates inflammasome activation during virus infection. Nat Immunol. (2013) 14:480-8. doi: 10.1038/ni.2563

86. Lu Q, Yokoyama CC, Williams JW, Baldridge MT, Jin X, Desrochers B, et al. Homeostatic control of innate lung inflammation by vici syndrome gene Epg5 and additional autophagy genes promotes influenza pathogenesis. Cell Host Microbe. (2016) 19:102-13. doi: 10.1016/j.chom.2015. 12.011

87. Castillo EF, Dekonenko A, Arko-Mensah J, Mandell MA, Dupont N, Jiang S, et al. Autophagy protects against active tuberculosis by suppressing bacterial burden and inflammation. Proc Natl Acad Sci USA. (2012) 109:E3168-76. doi: 10.1073/pnas.1210500109

88. Pu Q, Gan C, Li R, Li Y, Tan S, Li X, et al. Atg7 deficiency intensifies inflammasome activation and pyroptosis in pseudomonas sepsis. J Immunol. (2017) 198:3205-13. doi: 10.4049/jimmunol. 1601196

89. Cui SN, Chen ZY, Yang XB, Chen L, Yang YY, Pan SW, et al. Trichostatin A modulates the macrophage phenotype by enhancing autophagy to reduce inflammation during polymicrobial sepsis. Int Immunopharmacol. (2019) 105973. doi: 10.1016/j.intimp.2019.105973

90. Ip WKE, Hoshi N, Shouval DS, Snapper S, Medzhitov R. Antiinflammatory effect of IL-10 mediated by metabolic reprogramming of macrophages. Science. (2017) 356:513-9. doi: 10.1126/science. aal3535

91. Liang Q, Seo GJ, Choi YJ, Kwak MJ, Ge J, Rodgers MA, et al. Crosstalk between the cGAS DNA sensor and Beclin-1 autophagy protein shapes innate antimicrobial immune responses. Cell Host Microbe. (2014) 15:22838. doi: 10.1016/j.chom.2014.01.009

92. Chen M, Meng Q, Qin Y, Liang P, Tan P, He L, et al. TRIM14 inhibits cGAS degradation mediated by selective autophagy receptor p62 to promote innate immune responses. Mol Cell. (2016) 64:105-19. doi: 10.1016/j.molcel.2016.08.025

93. Eaglesham JB, Pan Y, Kupper TS, Kranzusch PJ. Viral and metazoan poxins are cGAMP-specific nucleases that restrict cGAS-STING signalling. Nature. (2019) 566:259-63. doi: 10.1038/s41586-019-0928-6

94. Sir D, Tian Y, Chen WL, Ann DK, Yen TS, Ou JH. The early autophagic pathway is activated by hepatitis B virus and required for viral DNA replication. Proc Natl Acad Sci USA. (2010) 107:4383-8. doi: 10.1073 /pnas.0911373107

95. Chan ST, Ou JJ. Hepatitis C virus-induced autophagy and host innate immune response. Viruses. (2017) 9:224. doi: 10.3390/v9080224

96. Shrivastava S, Raychoudhuri A, Steele R, Ray R, Ray RB. Knockdown of autophagy enhances the innate immune response in hepatitis $\mathrm{C}$ virusinfected hepatocytes. Hepatology. (2011) 53:406-14. doi: 10.1002/hep.24073

97. Shrivastava S, Devhare P, Sujijantarat N, Steele R, Kwon YC, Ray R, et al. Knockdown of autophagy inhibits infectious hepatitis $\mathrm{C}$ virus release by the exosomal pathway. J Virol. (2016) 90:1387-96. doi: 10.1128/JVI. 02383-15

98. Gratton R, Agrelli A, Tricarico PM, Brandao L, Crovella S. Autophagy in Zika virus infection: a possible therapeutic target to counteract viral replication. Int J Mol Sci. (2019) 20. doi: 10.3390/ijms20051048

99. Mateo R, Nagamine CM, Spagnolo J, Mendez E, Rahe M, Gale MJr, et al. Inhibition of cellular autophagy deranges dengue virion maturation. J Virol. (2013) 87:1312-21. doi: 10.1128/JVI.02177-12

100. Lai YC, Chuang YC, Chang CP, Lin YS, Perng GC, Wu HC, et al. Minocycline suppresses dengue virus replication by down-regulation of macrophage migration inhibitory factor-induced autophagy. Antiviral Res. (2018) 155:28-38. doi: 10.1016/j.antiviral.2018.05.002

101. Cao B, Parnell LA, Diamond MS, Mysorekar IU. Inhibition of autophagy limits vertical transmission of Zika virus in pregnant mice. J Exp Med. (2017) 214:2303-13. doi: 10.1084/jem.20170957

102. Shiryaev SA, Mesci P, Pinto A, Fernandes I, Sheets N, Shresta S, et al. Repurposing of the anti-malaria drug chloroquine for Zika Virus treatment and prophylaxis. Sci Rep. (2017) 7:15771. doi: 10.1038/s41598-01715467-6

103. Zhang S, Yi C, Li C, Zhang F, Peng J, Wang Q, et al. Chloroquine inhibits endosomal viral RNA release and autophagy-dependent viral replication and effectively prevents maternal to fetal transmission of Zika virus. Antiviral Res. (2019) 169:104547. doi: 10.1016/j.antiviral.2019.104547 
104. Duan X, Liu X, Li W, Holmes JA, Kruger AJ, Yang C, et al. Microrna-130a downregulates HCV replication through an atg5dependent autophagy pathway. Cells. (2019) 8:338. doi: 10.3390/cells 8040338

105. Pu J, Wu S, Xie H, Li Y, Yang Z, Wu X, et al. miR-146a Inhibits dengue-virusinduced autophagy by targeting TRAF6. Arch Virol. (2017) 162:3645-59. doi: 10.1007/s00705-017-3516-9

106. Fu Y, Xu W, Chen D, Feng C, Zhang L, Wang X, et al. Enterovirus 71 induces autophagy by regulating has-miR-30a expression to promote viral replication. Antiviral Res. (2015) 124:43-53. doi: 10.1016/j.antiviral.2015.09.016
Conflict of Interest: The authors declare that the research was conducted in the absence of any commercial or financial relationships that could be construed as a potential conflict of interest.

Copyright (c) 2020 Tao and Drexler. This is an open-access article distributed under the terms of the Creative Commons Attribution License (CC BY). The use, distribution or reproduction in other forums is permitted, provided the original author(s) and the copyright owner(s) are credited and that the original publication in this journal is cited, in accordance with accepted academic practice. No use, distribution or reproduction is permitted which does not comply with these terms. 